\title{
Zur spätglazialen Morphogenese der Emser Tomalandschaft
}

Hans W. Zimmermann

\section{Einleitung}

Das Rheintal oberhalb Chur ist ein beliebtes Studienobjekt der Geologie, denn hier finden sich die wohl interessantesten Bergsturzmassen der Alpen. Daher haben sich schon eine größere Zahl von Autoren mit diesem Gebiet beschäftigt, zuletzt Nabholz (1954), Remenyik (1959) und Pavoni (1968). Die Zahl der Deutungsversuche der geomorphologisch wichtigen Vorgänge in dieser Landschaft ist entsprechend groß, und manche Punkte sind bislang unabgeklärt geblieben oder nur unbefriedigend gedeutet worden. Mit der Veröffentlichung einiger Beobachtungen aus den letzten Jahre soll hier ein weiterer Beitrag zur Kenntnis dieses Gebietes geleistet werden.

Zuerst sei das bisher gewonnene Wissen über das östlich an den Flimser Bergsturz anschließende Trümmer- und Terrassengebiet kurz zusammengefaßt:

In einer umfangreichen Publikation hat Remenyik (1959) die verhandene Literatur durchgearbeitet und mit den eigenen Untersuchungsergebnissen verglichen. Seine Resultate seien, soweit sie unser Gebiet betreffen, kurz ausgezogen: Zwei ältere Bergstürze (Flims und Silberegg/Goldene Sonne) sind gefolgt van einer fluviatilen Erosionsphase. Dann folgen zwei kleinere Stürze, Kunkelspaß/Reichenau und Ems/Felsberg; sie trennen ein Becken von Bonaduz bis Cazis ab, dessen Stausee von Schotter aufgefüllt wird. Der Rheingletscher überfährt im Churer Stadium die ganzen Schuttmassen und hinterläßt Moränenreste. Hierauf tieft sich der Rhein unter Bildung von Erosionsterrassen bis auf das heutige Niveau ein.

Pavoni (1968) überarbeitete das Gebiet und kam zu einigen Modifikationen. Ihm fiel auf, daß die Schotter im Bonaduzer Becken zum Teil völlig unnatürliche Neigungen aufweisen. Nun nimmt er an, daß Bergsturzmaterial und Schotter auf den Ablagerungen eines spätglazialen Sees liegen; der letzte Bergsturz von der Westseite des Kunkelspasses habe dann bei seinem Aufprall die vorhandene Beckenfüllung gestaucht und weggeschoben, ja zum Teil zum «Wegfließen» gebracht.

\section{Geologische Übersicht}

Das Rheintal oberhalb Chur weist zwei grundverschiedene Talhänge auf. Die Nordseite wird zum größeren Teil durch die mehr oder weniger hangparallel aufsteigenden Kalkbänke des helvetischen Autochthons gebildet, die im Calanda gipfeln. Wegen der starken Unterschneidung durch den eiszeitlichen Rheingletscher sind die meisten Schichten von ihren Wurzeln abgetrennt, so daß die gipfelseitigen Schichtköpfe heute als drohende Felsabstürze bandförmig über dem Tal durchziehen (daher auch die Teilverlegung des Dorfes Felsberg im 19. Jahrhundert). Nördlich von Ems ist die Durchtrennung des Autochthons sogar vollständig, so daß über eine größere Strecke das Aarmassiv aufgeschlossen ist; aus der Ferne fallen die grünen Epidoth-Chlorit-Schiefer sofort auf. Die Hydrographie des Hanges ist entsprechend, ständige Gewässer fehlen, der fluviatile Abtrag ist gering; nur selten geht ein Murgang nieder und baut an den wenigen Schuttkegeln weiter (Abb. 1). Durch Frostsprengung wird dagegen ständig Nachschub für große Schutthalden geliefert. Heute sind meist nur die obern Partien aktiv, während die tieferen Schuttmassen fossilisiert von Eichen-Föhren-Wald überzogen sind.

Der südliche Talhang wird vorwiegend von mittelpenninischen Bündnerschiefern aufgebaut. Er ist regelmäßig dicht von Fichten bestanden, was auf die leichte Verwitterbarkeit des Untergrundes und die bessere Wasseraufnahme hinweist. $\mathrm{Da}$ das Meteorwasser aber nicht in die Tiefe sickern kann, ist der Hang von Tobeln durchfurcht, an deren Fuß sich gewaltige Schwemmfächer ausbreiten (Abb. 2). Durch das ständige Wachstum reichen diese heute sogar weit in die Tobel hinauf, so daß ihre Spitzen 300 bis $400 \mathrm{~m}$ über den Talboden aufragen. Das Material der Kegel ist so merkwürdig, daß es noch gesondert besprochen werden wird.

Der Talboden ist van wechselnder Breite und besteht unterhalb Ems aus recht gut gerundeten Schottern des Rheins; oberhalb Ems wird er streckenweise durch eine Erosionsfläche in Bergsturzschutt gebildet. Aus diesem Talboden ragen zuerst nur unbedeutende (Chur, Felsberg), dann mächtige Hügel 
(Ems) aus Kalktrümmern auf, die sich vor Reichenau zu einer Barriere quer durch das Tal fast schlieBen.

\section{Geomorphologische Übersicht}

zu Figur 1 und LK Blatt 1195 Reichenau

Die großen geologischen Unterschiede auf relativ kleinem Raum drücken sich noch stärker im Formenschatz der Landschaft aus, da die petrographischen Faktoren noch durch solche der Exposition verstärkt werden.

Der Sonnenhang des Tales zeichnet sich durch das fast völlige Fehlen fluviatiler Formelemente aus; Felsbänder, die rückwittern, aktive und fossile Schutthalden sowie einige sehr steile $(25 \%)$ und wenig tiefgründige Schuttkegel machen einen direkt ariden Eindruck.

Der Schattenhang hingegen zeigt Tobel, Runsen und Racheln, die zum Teil vegetationslos sind, sowie gewaltige Schwemmkegel, also ein Schulbeispiel für fluviatile Landschaftsformung.

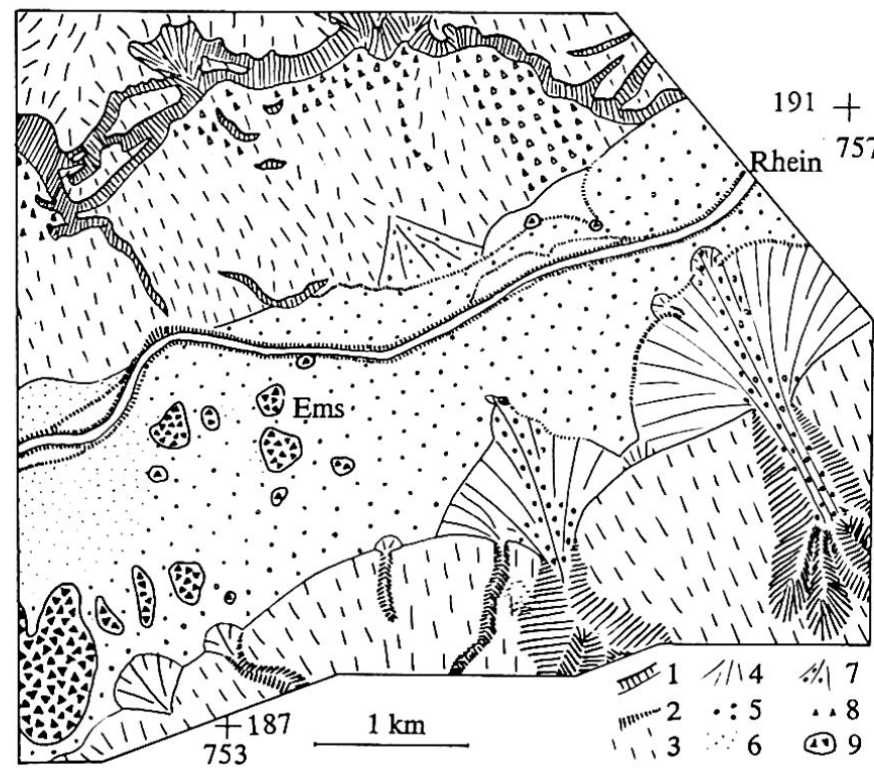

Figur 1. Geomorphologische Kartenskizze des Rheintals bei Ems. 1 Schichtköpfe der unterschnittenen Kalkschichten des helvetischen Autochthons, durch Frostsprengung zurückwitternd. 2 Fluviatil (durch Seiten-oderTiefenerosion) geschaffene Steilhänge; Rutsch- und Sackbewegungen; dazu gehören Prallhänge von Mäandern, Terrassenhänge, Tobelwände. 3 Der subaerischen Denudation unterworfene Hänge; Bodenkriechen und Abschwemmung. 4 Schwemmkegel aus Feinmaterial, sandigsiltig. 5 Schotterterrassen des Rheins (Hauptniveau). 6 Erosionsterrassen des Rheins in Bergsturzschutt (Hauptniveau). 7 Schuttkegel aus grobem Material (Blöcke, Geröll, Kies), meist Murgangmaterial. 8. Schutthalden, soweit heute noch aktiv. 9 Tomas, Hügel aus Bergsturzmaterial
Der Talboden ist durchsetzt mit einem fremdartigen Element, den Tomas (sutselvisch tuma, vom lat. tumulus, Hügel); diese merkwürdig geformten Bergsturzmassen verraten vorerst nicht, ob sie Akkumulations- oder Erosionsformen sind.

In den folgenden Abschnitten werden nun einige Landschaftselemente und Aufschlüsse besprochen, die sich als Schlüsselpunkte für das Verständnis der Morphogenese dieses Gebietes erwiesen haben.

\section{Die Schwemmkegel}

Van Ems bis zur Luziensteig sind die gewaltigen Aufschüttungen auf der rechten Seite des Rheintales das charakteristische Landschaftselement. Ihre Oberflächenneigung ist recht groß, beginnt bei etwa $13 \%$ in tieferen Lagen und steigt auf bis 35\% an den Spitzen der Kegel. Die Höhe dieser Kegel ist nicht ganz sicher zu bestimmen, da die Spitzen in Tobel mit einer Füllung von Murgangschutt übergehen. Doch auch Näherungswerte sind immer noch eindrücklich genug:

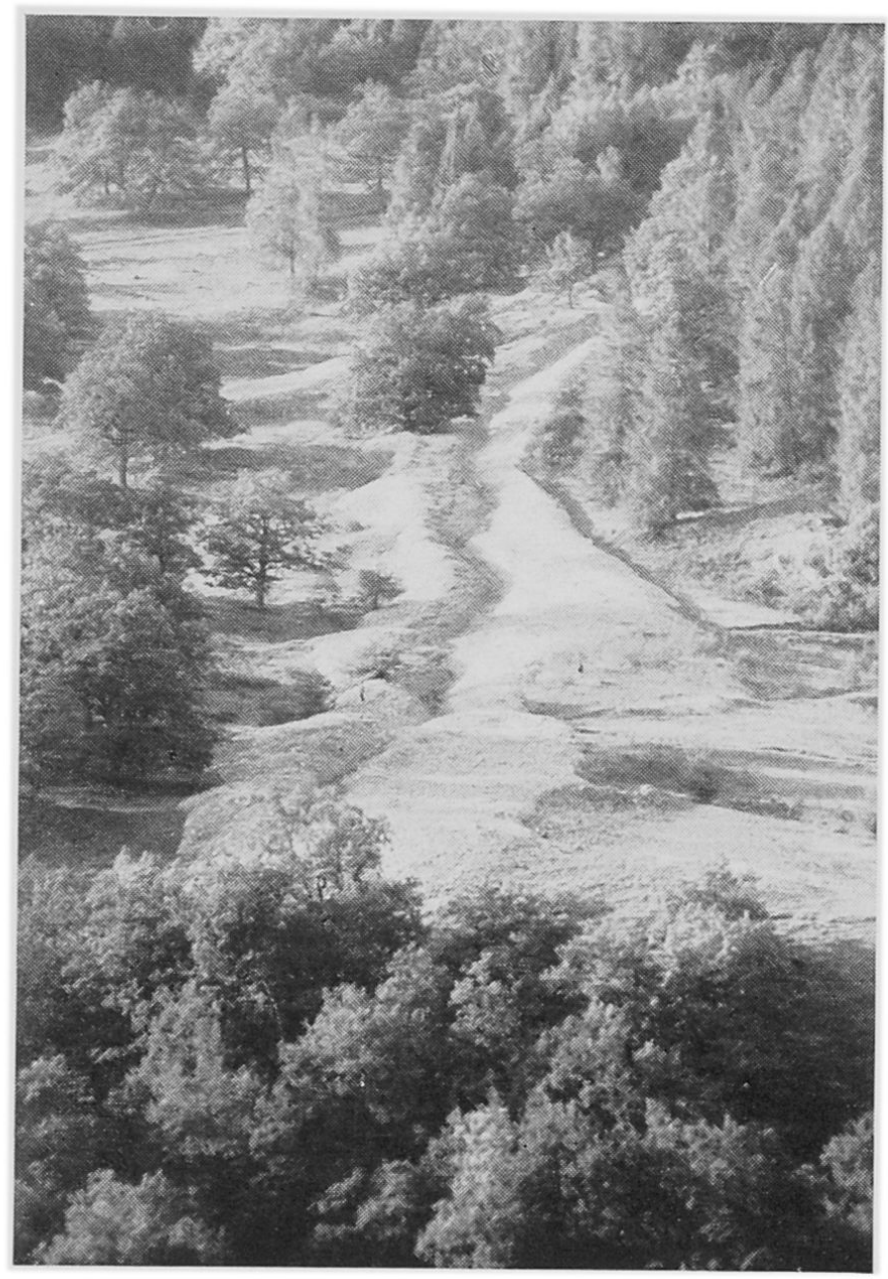

Abbildung 1. Murgang vom Sommer 1970 im Eichwald $W N W E m s$, eine der seltenen fluviatilen Erscheinungen an der nördlichen Talflanke 


$\begin{array}{lrrr}\text { Ausgangstal } & \text { Basis } & \text { Spitze } & \begin{array}{l}\text { Höhen- } \\ \text { differenz }\end{array} \\ \text { Val Mulin (Ems) } & 580 & 750 & 170 \mathrm{~m} \\ \text { Val Parghera } & 570 & 940 & 370 \mathrm{~m} \\ \text { Altenschutz (W Chur) } & 570 & 790 & 220 \mathrm{~m} \\ \text { Kaltbrunnertobel (N Chur) } 560 & 1000 & 440 \mathrm{~m} \\ \text { Scaläratobel (Fürstenwald) } 560 & 1040 & 480 \mathrm{~m}\end{array}$

Der recht beträchtliche Neigungswinkel (über $7^{\circ}$ ) steht nun in keiner Beziehung zum Material, das den Hauptkörper der Schwemmkegel aufbaut. Die Spitzen der Kegel sowie oberflächliche Streifen, die bis in die Talsohle reichen und an Vegetation und Landnutzung leicht erkennbar sind, bestehen aus holozänem, zum Teil rezentem Schutt von Murgängen und widerspiegeln damit die heutigen Abtragungsvorgänge.

Darunter aber folgen mindestens $8 \mathrm{~m}$ (tiefster beobachteter Aufschluß) sandig-siltiges, manchmal lehmiges Material mit einzelnen kleinen Steinen; der Farbe nach enthält es sehr wenig organische Substanz, dem Verwitterungsgrad nach aber könnte es abgeschwemmter Boden sein. Eine Folgerung läßt sich aus diesem Befund sicher ziehen: Diese Aufschüttung paßt nicht in unser gemäßigtes Klima. Die obersten zwei Meter des Feinmaterials, bereits stellenweise stärker mit Steinen durchsetzt, führen lokal fossile Schnecken. Drei Stellen konnten ausgebeutet werden:
A Masans, Neubau des Evangelischen Altershei- mes, $620 \mathrm{~m}$
B Chur, Masanserstraße 100, $585 \mathrm{~m}$
C bei Ems, 755 650/189 150, $580 \mathrm{~m}$
In die gleiche Tabelle werden der Einfachheit hal- ber noch die Funde von der Tuma Casti in Ems ge- fügt:
D Ems, 751 250/189 140, $610 \mathrm{~m}$

Art

1. Lymnaea (Galba) truncatula (Müll.) Kleine Schlammschnecke

2. Cochlicopa lubrica (Müll.) Glatte Achatschnecke

3. Perforatella cf. rubiginosa (A. Schm.) Rotbraune Laubschnecke

4. Arianta (Helicigona) arbustorum (L.) Gefleckte Schnirkelschnecke

5. Columella edentula (Drap.) Zahnlose Windelschnecke

6. cf. Monarcha cartusiana (Müll.) Kartäuserschnecke

7. Vitrea cristallina (Müll.) Kristall-Glanzschnecke

8. Helix pomatia L. Weinbergschnecke

9. Gonyodiscus (Discus) rotundatus (Müll.) Gefleckte Diskusschnecke

10. Candidula (Helicella) unifasciata (Poir.) Einstreifige Heideschnecke

11. Aegopinella (Retinella) pura (Alder) Kleine Glanzschnecke

12. Aegopinella (Retinella) nitens (Mich.) Weitmündige Glanzschnecke

Unsicherheiten in der Bestimmung sind auf oft starke Beschädigungen an den Gehäusen zurückzuführen.

Die gefundenen Arten kommen alle noch heute in der Gegend vor, doch gehören sie nicht einem einheitlichen Biotop an. Neben einer Wasserschnecke (1), die noch in kleinsten Rinnsalen auftritt, sind Ufer- und Auebewohner (2-7) sowie Arten eher trockener waldig-steiniger Standorte vorhanden (8-12).

\begin{tabular}{|c|c|c|}
\hline A & B & C \\
\hline $\mathbf{x}$ & & $x$ \\
\hline $\mathrm{X}$ & & $x$ \\
\hline \multirow[t]{5}{*}{$\mathrm{X}$} & $x$ & $x$ \\
\hline & & $\mathbf{x}$ \\
\hline & & $\mathrm{x}$ \\
\hline & & $\mathrm{x}$ \\
\hline & & \\
\hline $\mathrm{x}$ & $\mathrm{x}$ & \\
\hline $\mathrm{x}$ & $\mathrm{x}$ & \\
\hline $\mathrm{x}$ & $\mathrm{x}^{1}$ & $\mathbf{X}$ \\
\hline & $x$ & \\
\hline
\end{tabular}

$\mathrm{X}$
Die Arten 2, 4, 5 und 7 kommen auch in den eiszeitlichen Lößen der Schweiz vor (Gouda 1962, 150), sind also mit ihrer Klimaunempfindlichkeit für eine Datierung nicht brauchbar. Interessanter ist die Art 9: Ihr erstes postglaziales Auftreten wird von Stehlin (1941) aus der frühmesolithischen Station Balm bei Günsberg SO erwähnt, welche wiederum von Wyß (1968) auf etwa 7800 v. Chr. datiert wird; zusammen mit andern eher wärmeliebenden Schnecken und einer Säugerfauna, die aus alpinen 
und gemäßigten Vertretern gemischt ist, entsteht dort der Eindruck, daß gerade der Einbruch der postglazialen Klimabesserung erfaßt worden ist. Die eher wärmeliebende Molluskenfauna in den feinkörnigen Deckschichten der Schwemmkegel bei Chur dürften etwa in der gleichen Zeit der postglazialen Klimabesserung entstanden sein.

Die weitere Formung der Schwemmkegel nimmt kein großes Ausmaß mehr an. Immerhin ist noch eine Feststellung $\mathrm{zu}$ treffen:

Der Rhein hat am Fuß der Kegel seine Spuren in Form einiger Prallhänge zurückgelassen, bevor er sich in seiner jetzigen Lage in den Talboden einschnitt (Abb. 2).

An solchen Stellen läßt sich heute am besten sehen (Fig. 1), was für einen Umfang die seitherigen Schüttungen angenommen haben, denn die entsprechenden Ablagerungen heben sich deutlich von ihrer Umgebung ab; sie bilden nur noch einen Streifen über die Kegeloberfläche und werden noch heute von den Bächen benutzt.

Die relative Schonung der übrigen Kegeloberfläche findet auch darin ihren Ausdruck, daß im Mittelalter eine große Zahl von Terrassenäckern angelegt worden sind, die auch heute noch das Kleinrelief bestimmen (Abb. 2).

\section{Die Tomas von Ems}

a) Tuma Casti

Dieser Hügel liegt gleich W des Dorfzentrums von Ems. Auf der Süd- und auf der Nordseite finden sich je eine heute aufgelassene Grube.

Jene auf der Südseite zeigt durchwegs feine Bergsturzbrekzie, da die dort abgelagerten Blöcke zum Teil im Schichtverband durch und durch zerrüttet worden sind, so daß sie mit dem Pickel leicht abgebaut werden können. Rätselhaft ist, daß so zerrüttete Felsmassen direkt an der Oberfläche liegen: Offenbar ist ein beträchtliches $\mathrm{Ma} ß$ an hangendem Material seit der Ablagerung der Sturzmassen verschwunden. Auf ursprünglich von den heutigen abweichende Verhältnisse deuten auch einige leichte Kalkausscheidungszonen hin, die auf einen in Dachhöhe des heutigen Dorfes liegenden Grundwasserspiegel hinweisen. Aus beidem muß geschlossen werden, daß diese Toma - oder wenigstens die Südseite - einen Erosionsrest darstellt.

Auf der Nordseite sind die Verhältnisse komplizierter, wie Figur 2 zeigt.

Die Basis besteht wiederum aus Bergsturzschutt. Die Blöcke - auch hier zum Teil noch im Schichtverband liegend - sind etwas weniger stark zerrüttet als auf der Südseite des Hügels. Merkwürdig ist auch hier die Oberfläche der Sturzmasse; bei jedem Sturz bleiben solide große Blöcke zuoberst liegen,

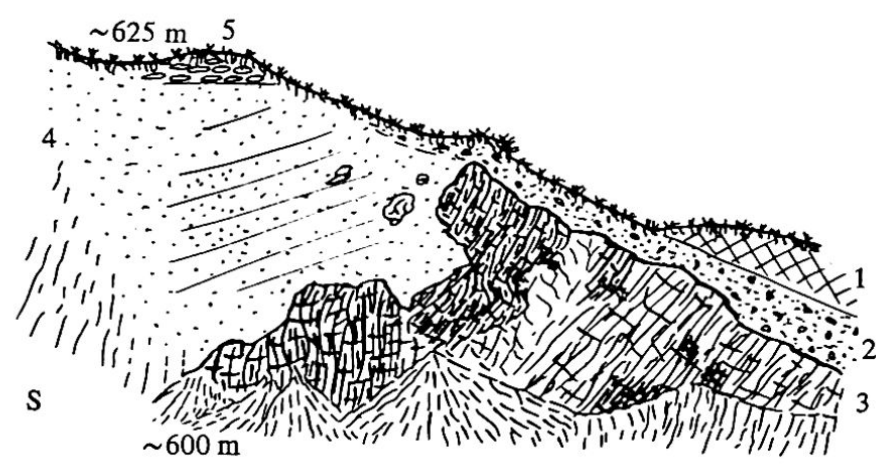

Figur 2. Westwand der Grube auf der Nordseite der Tuma Casti, Ems. 1. Aufschüttung (Grubenabraum. 2. Abschwemmaterial (Hangschutt), schnekkenführend. 3. Bergsturzschutt, dunkle und hellere Malmkalke, stark zerrüttet. 4. Sandig-lehmige Masse mit leicht nach Süden geneigter Schichtung, Eisrandbildung. 5. Kameschotter, grob, sehr schlecht gerundet

wie dies in einer Grube an der Westseite der Tuma Padrusa noch gut beobachtet werden kann; hier aber sind Zerrüttungsmassen an der Oberfläche, und der Schichtverband ist diskordant abgetrennt. Ein Brocken dunklen Malmkalks steht sogar leicht überhängend. Dies weist darauf hin, daß weder ursprüngliche Oberfläche noch ursprüngliche Lage heute erhalten sind.

Über dem Bergsturz liegen bis $20 \mathrm{~m}$ lehmige Sande; sie enthalten gegen oben zu immer häufiger kleine

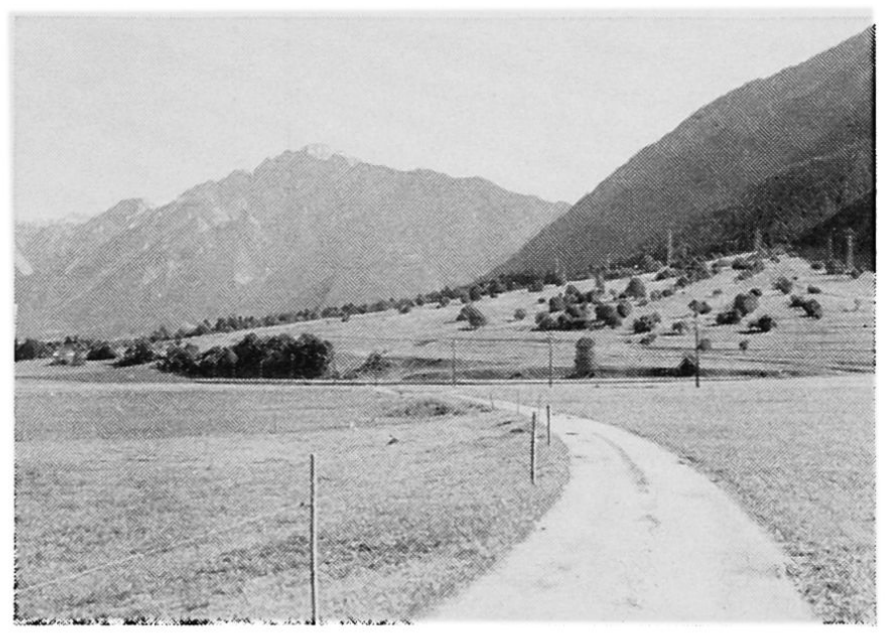

Abbildung 2. Der große Schwemmkegel am Fuße des Val Parghera zwischen Chur und Ems. Sichtbare Höhendifferenz $140 \mathrm{~m}$. Der Waldstreifen, der die Silhouette gegen hinten unterstreicht, bedeckt die Zone mit Murgangschutt. Vorne Unterschneidung des Kegels durch den Rhein an der Wende jüngere Dryaszeit/Alleröd; am Ende der Unterschneidung links sekundärer junger Schuttkegel, der das Ausmaß der holozänen Aufschüttung ins richtige Verhältnis rückt. Ackerterrassen sind am Schattenwurf erkennbar 


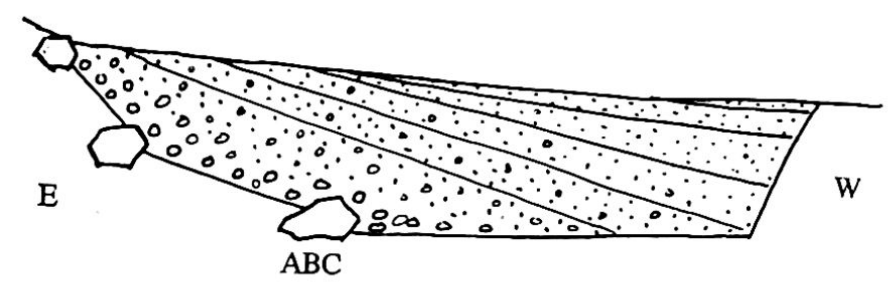

Figur 3. Querschnitt durch die Kameterrasse $S$ der Station Reichenau. Auffällig ist das Ansteigen und Auskeilen der Schichten nach Osten. A, B, C drei hintereinanderliegende große Bergsturzblöcke mit glazialer Politur und Schrammen; dazwischen lag noch ein Findling

meist kristalline Steine. Sie sind sicher nicht im Zusammenhang mit dem Bergsturz entstanden, denn ihr ortsfremdes Material liegt auf einer Erosionsoberfläche. Die Schichtung der Masse ist nur schwach erkennbar, aber sie zeigt deutlich eine Neigung nach Süden, also verstelit wie der darunter liegende Bergsturzschutt. Für diese Kippung kommen drei Ursachen in Frage:

1. Abschmelzen von Toteis unter dem Bergsturz;

2. Unregelmäßige Setzung der Sturzmasse bei der Uberdeckung durch das Eis des Churer Stadiums; 3. Verstellung durch Eisdruck von der Seite.

Diese dritte Ursache erscheint uns am wahrscheinlichsten, da damit gleichzeitig die Erosionsoberfläche erklärt werden kann.

Während die Hügelkuppe mit der Kapelle aus Bergsturzmaterial besteht, findet sich an einer kleinen Schulter auf der Nordseite über den Sanden eine Lage sehr groben, schlecht gerundeten Schotters; kaum kantengerundetes Kristallin muß schon fast

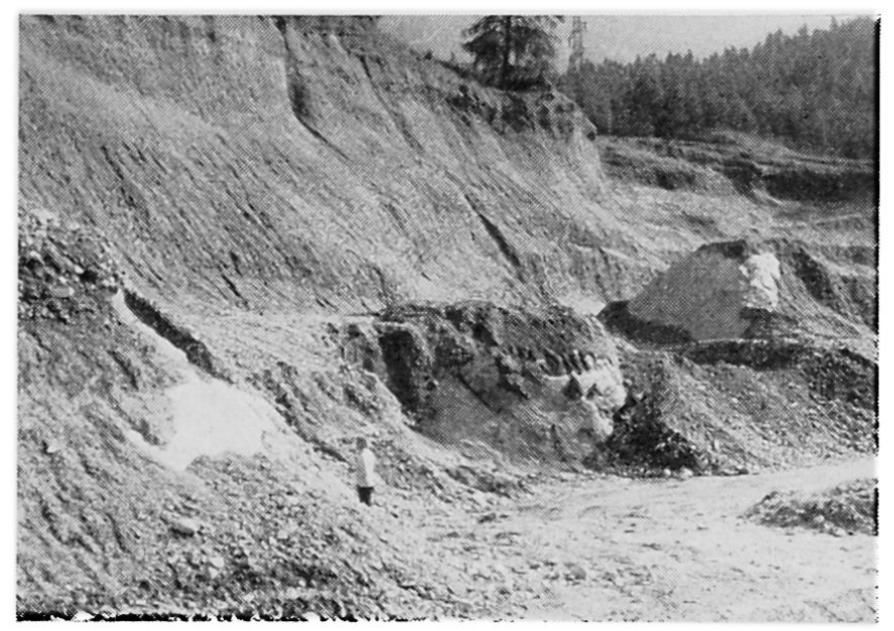

Abbildung 3. Kieswerk Reichenau. An der Ostwand erscheinen unter den Kameschottern drei gewaltige vom Eis gerundete, geschrammte und geschliffene Bergsturzblöcke aus Malmkalk; vorne links Block $A$, nach hinten folgend $B$ und $C$ des Textes als Obermoräne hierhergekommen sein. Es dürfte sich wie bei den Sanden darunter um Kamebildungen aus der Toteisphase des Churer Stadiums handeln. Eine änhliche Beobachtung von Schottern, wenn auch in etwas tieferer Lage, ist von Prof. J. Cadisch (mündl. Mitteilung in Remenyik 1959, 222) an der Ostseite des Walserbühls am westlichen Stadtrand von Chur gemacht worden.

Die Hänge der Emser Tomas sind, wie in allen Aufschlüssen sichtbar, von einem Mantel aus Hangschutt umgeben. Der Rhein hat, als er die Emser Terrasse benützte, die Hügelbasis unterspült und so ein ständiges Nachbröckeln der Hänge hervorgerufen. Hier auf der Nordseite der Tuma Casti ist wegen des feinen Materials aber eine Art Abschwemmdecke entstanden. Vor ihrer Fixierung durch die Vegetation, die auf das Ende der Hangunterspülung folgte, wurden noch einige Mollusken mit einsedimentiert; aber wegen der ständigen Kriech- und Rutschbewegung sind meist nur Trümmer aufzufinden. Immerhin konnten (Tabelle 5, Kolonne D) zwei bis drei Arten bestimmt werden, die in den Rahmen der andern Molluskenfunde passen.

\section{b) Tuma Falveng}

An diesem Hügel findet sich $\mathrm{m}$. W. der einzige frei gelagerte Findling im Raume Ems, der eine glaziale Überfahrung der Tomas dokumentiert; er wurde mir freundlicherweise von Herrn Dr. E. Kirchen (Chur) gezeigt. In der Bodenschicht sind dagegen oft kleine Blöcke und Kiesel ortsfremden Materials anzutreffen, doch könnte ein Teil davon auch von früherer Bautätigkeit stammen. Die Ansicht von Remenyik (1959, 220), daß eine zusammenhängende Moränendecke den Hügel umgebe, kann mangels Aufschlüssen von genügender Größe nicht bestätigt werden. Handelt es sich vielleicht teilweise um Abschwemmaterial wie an den Tumas Casti und Padrusa?

\section{Die Kameterrasse von Reichenau}

Südöstlich der Station Reichenau-Tamins der Rätischen Bahn liegt eine gewaltige Toma, Ils Aults. An ihrer Nordwestseite findet sich eine Terrasse angefügt, deren Material durch das Kieswerk Reichenau ausgebeutet wird.

Der Hang im Bergsturzmaterial fällt kesselförmig von $\mathrm{E}$ und von $\mathrm{S}$ her ein und verschwindet unter einer auffälligen Schottermasse (Fig. 3). Diese läßt nur selten eine Schichtung erkennen, welche in den tieferen Teilen mit 20 bis $25^{\circ}$ gegen die Station Reichenau einfällt, also steiler als die geneigte Oberfläche der Terrasse des Plong Vaschnaus.

Liegend besteht die Ablagerung aus relativ groben, gut gewaschenen Schottern, geht dann aber in im- 
mer feinere Fraktionen über; weit mehr als die Hälfte der Ablagerungsmächtigkeit wird durch kiesig-sandige, fast strukturlose Massen gebildet. Das Gesamte macht den Eindruck eines einzigen überdimensionierten "graded bedding".

Das Auskeilen und die Schiefstellung der Schichten deutet an, daß die Ablagerung auf langsam abschmelzendes Toteis erfolgte, es handelt sich also um eine typische Kamebildung. Ähnliche Schiefstellungen von fluviatilen Sedimenten sind im ganzen Gebiet häufig (Pavoni 1968), dürften aber auch andere Ursachen haben (z. B. Deltabildungen).

Die Höhe der Terrasse, am obern Rand etwa 675 m, zeigt auch, daß eine heute nicht mehr vorhandene Stauursache zu dieser Kamebildung geführt haben muß. Der Haupttalboden, die Emser Terrasse, liegt etwa $60 \mathrm{~m}$ tiefer, also muß er auch mit Eis gefüllt gewesen sein, damit der Wasserabfluß so stark behindert werden konnte.

Interessant sind die Masse des Bearbeitungsgrades der Gerölle. Es wurden drei Proben untereinander und mit solchen aus dem Mittelland verglichen.

Material: Kalkgerölle von 30-80 mm Länge.

Probelage:

A Kieswerk Reichenau, SE-Ecke (in Fig. 3 hinter den Blöcken A, B, C), 3-5 m über der Bergsturzmasse (Liegendes);

B Kieswerk Reichenau, $10 \mathrm{~m}$ über dem Bahnhof (Hangendes);

C Schotterbank im Vorderrhein W Schloß Reichenau.

Ergebnisse:

Abplattungsindizies:

A 2,39 Wert relativ hoch, offenbar petrographisch bedingt;

B 2,83 Abplattung stärker, also Fluß etwas weniger wild;

C 3.36 Abplattungsmaximum unter den heutigen Verhältnissen, die Wasserführung ist a!so heute ganz bedeutend regelmäßiger als zur Zeit der Kamebildung, so daß die Bearbeitung durch Treibsandschliff zum Charakteristikum wird.

Zurundungsindizes:

C 260 recht gering im Vergleich zu eiszeitlichen Sandern im Mittelland; dort ergeben sechs Messungen Werte zwischen 270 und 300 (Zimmermann 1963, 32), bei einem Esker - einer inglazialen Bildung - 240. Die Zufuhr von frischem Bergsturzmaterial von den Prallhängen des Vorderrheins in der Schlucht durch den Flimser Bergsturz ist also stark spürbar.

B 195 Der tiefe Wert zeigt, daß Bergsturzmaterial ohne großen fluviatilen Transport hierher-
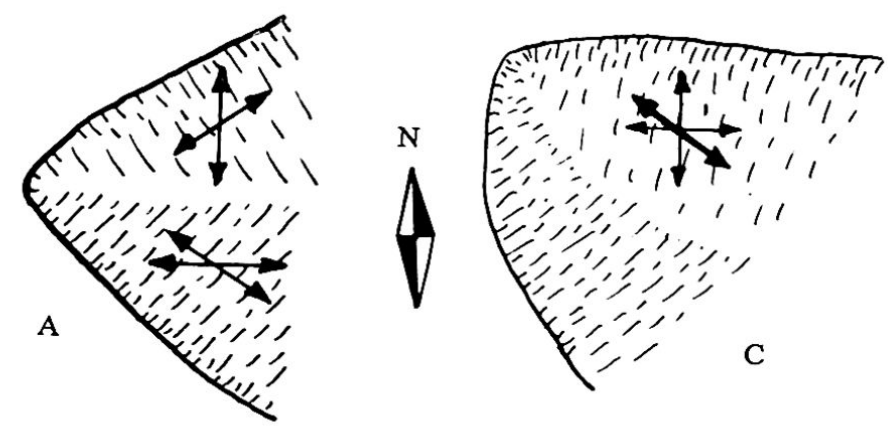

Figur 4. Richtung der Gletscherschrammen auf zwei Bergsturzblöcken am Grund der Kiesgrube Reichenau; die wechselnden Richtungen zeigen eine Bewegung der Blöcke an

gelangt ist. Noch deutlicher wird diese Feststellung durch die Betrachtung der einzelnen Zurundungsklassen: Zwischen $\mathrm{O}$ und 100 finden sich $35 \%$ der "Gerölle» oder besser Trümmerstücke; ein zweites Maximum in der Häufigkeitsverteilung zwischen 200 und 300 mit $25 \%$ der Gerölle, entspricht dem heutigen Flußschotter; es scheint, als habe der Gletscher auch schon vorhandenen Flußschutt aufgearbeitet und mit seinem Schmelzwasser in dem Kame wieder sedimentiert. Damit bestätigt sich möglicherweise auch die Feststellung von Remenyik (1959, 226), daß es zwischen den Niedergängen verschiedener Bergstürze zu Schotterbildungen gekommen sei.

A 190 Praktisch gleicher Wert wie bei B, also sind die Herkunftsverhältnisse ungefähr dieselben.

Einregelung:

A NNE Die Fließrichtung war schön parallel zum Hang; da $72 \%$ der Gerölle innerhalb der $30^{\circ}$-Abweichung liegen, kann angenommen werden, daß der Fluß zwischen Talhang und Gletscher nicht viel Spielraum hatte, sondern beinahe kanalisiert dahinfloß. Zum Vergleich: acht Proben in den großen Schotterfluren um Solothurn ergaben Werte zwischen 31 und 52\%.

Unter diesen Kameschottern liegen zum Teil riesige Bergsturzblöcke des helvetischen Malms. Die Oberfläche der drei größten Brocken (Abb. 3) ist glazial poliert und geschrammt. Eine nähere Betrachtung lohnt sich (Fig. 4): Der nördlichste der drei Blöcke, A, zeigt zwei Flächen; jene nach NW mit etwa $35^{\circ}$ Neigung zeigt zwei Schrammenrichtungen, die steilere SW-Seite ebenfalls, aber nicht dieselben. Beim südlichsten Block, C, war durch den Abbau erst die $45^{\circ}$ steile Nordseite freigelegt; sie zeigte gar drei 


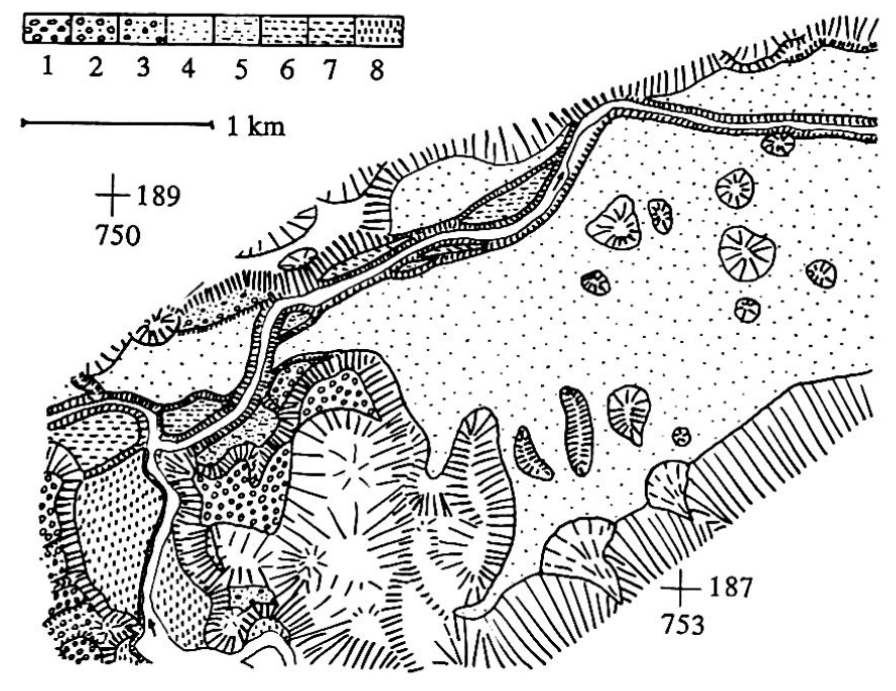

Figur 5. Die Rheinterrassen zwischen Bonaduz und Ems. Die Ziffern bezeichnen die verschiedenen Niveaus; Erklärung im Text

Schrammrichtungen. Das Eis hat also diese gewaltigen Blöcke zu bewegen vermocht; weniger grobes Material wird daher wohl in großen Mengen dem glazialen Abtrag anheimgefallen sein.

Zwischen den Blöcken A und B lag noch ein über meterlanger Findling aus Epidoth-Chlorit-Schiefer, der auf den Vorderrheingletscher hinweist.

\section{Die Rheinterrassen}

\section{Zu Figur 5 und 6}

Eine dominierende naturgeographische Einheit ist der weite Talboden von Ems, der hier eigentlich als Terrasse zu bezeichnen ist. Neben ihm werden die flächenmäßig unbedeutenden höheren und tieferen Terrassenreste kaum beachtet. Dabei lassen sich an ihnen recht interessante Feststellungen machen. Die Festlegung bestimmter Niveaus begegnet allerdings einigen Schwierigkeiten, da die Oberflächen der Terrassen nicht eben, sondern von ehemaligen Flußläufen zerfurcht sind; bei großen Flächen stört dies nicht, bei kleinen aber wissen wir nie, ob der obere Teil eines Gleithanges oder die Mulde eines Mäanders erhalten geblieben ist; die folgende Darstellung muß daher mit den entsprechenden Vorbehalten betrachtet werden.

Im Prinzip sind acht Terrassenniveaus sicher unterscheidbar; drei sind älter als der Haupttalboden, vier dagegen sind später entstanden. Sie seien hier kurz besprochen:

1. Akkumulationsniveau aus der Toteisphase des Churer Stadiums des Vorderrheingletschers. Material: Schotter und Sande, Stau- und Kamebildungen mit Toteistektonik. Die Oberfläche der Kameschot- ter $\mathrm{S}$ der Station Reichenau lag ursprünglich auf etwa $670 \mathrm{~m}$, dann sank sie trotz fortwährender Einschüttung auf $650 \mathrm{~m} \mathrm{ab}$, da das Eis darunter wegschmolz; mit ähnlichen Erscheinungen muß überall im Gebiet des Banaduzer Beckens gerechnet werden. Das Akkumulationsniveau ist also nicht überall gleich alt, sondern an den höhern Rändern älter als in den Mulden und auf dem Schwemmkegel des Vorderrheins W Bonaduz. Ein Gefälle des akkumulierenden Flusses ist daher nicht festlegbar. Die Schotter an der Tuma Casti in Ems sind nur mangels besserer Lösung hier mit angeschlossen.

2. Trompetentalähnliches oberstes Erosionsniveau: Gefälle 10\%. Senkt sich NW Bonaduz und E Rhäzüns in das Akkumulationsniveau ein, liegt $\mathrm{E}$ der Station Reichenau bereits $15 \mathrm{~m}$ tiefer und endet hier. Nicht zu verwechseln mit einem echten Trompetental, dessen Gefälle geringer als das des Akkumulationsniveaus ist.

3. Zweites Erosionsniveau; Gefälle 11,5\%o. Breiter als das vorherige; ebenfalls E Tamins/Reichenau endend.

4. Hauptniveau, Emser Terrasse. Oberer Teil Erosionsterrasse in Bergsturzschutt und Schotter, Gefälle $12 \%$; diese Oberfläche zieht am westlichen Dorfrand von Ems unter die Akkumulationen des unteren Teils (Gefälle 9\%).

Das Auffällige an diesen Niveaus im Raume Tamins ist die stete Zunahme des Terrassengefälles von oben nach unten. Daraus können wir schließen, daß eine lokale Erosionsbasis vorhanden war, die rasch tiefergelegt worden ist; sie muß wenige $100 \mathrm{~m}$ NE der Station Reichenau gewesen sein. Zwei Möglichkeiten kommen dafür in Betracht, nämlich Toteis und Bergsturzmaterial. Da das Akkumulationsniveau starke Toteistektonik zeigt, dürfte die Annahme eines Toteiskörpers auch unterhalb des Bonaduzer Beckens im Raume von Ems richtig sein, denn darauf weisen auch die merkwürdigen Schotter an der Tuma Casti hin. Andererseits ist das Hauptniveau eindeutig im Bergsturzschutt des Engpasses E Tamins fixiert, womit die stauende Ursache gegeben ist. Was von beidem für die Niveaus 2 und 3 zutrifft, kann nicht belegt werden; die Breitentwicklung des Niveaus 3 läßt eine solidere Barriere als Eis vermuten.

Das über weite Strecken verfolgbare große Gefälle erlaubt noch eine weitere, etwas spekulative Überlegung: Wo war denn die Erosionsbasis, nämlich das Ufer des Bodensees, damals? Lassen wir das Gefälle langsam auf $4 \%$ zurückgehen, so wird etwa $30 \mathrm{~km}$ später, das heißt in der Gegend von Sargans, der (heutige) Seespiegel von $396 \mathrm{~m}$ erreicht.

Die Breite der Terrasse ist völlig unnatürlich, die allgemeine Tendenz der Tiefenerosion ist durch eine große Seitenerosionsphase ersetzt. Eine solche Erscheinung ist normalerweise durch einen Stau verursacht; als Verursacher kommt materialmäßig nur 


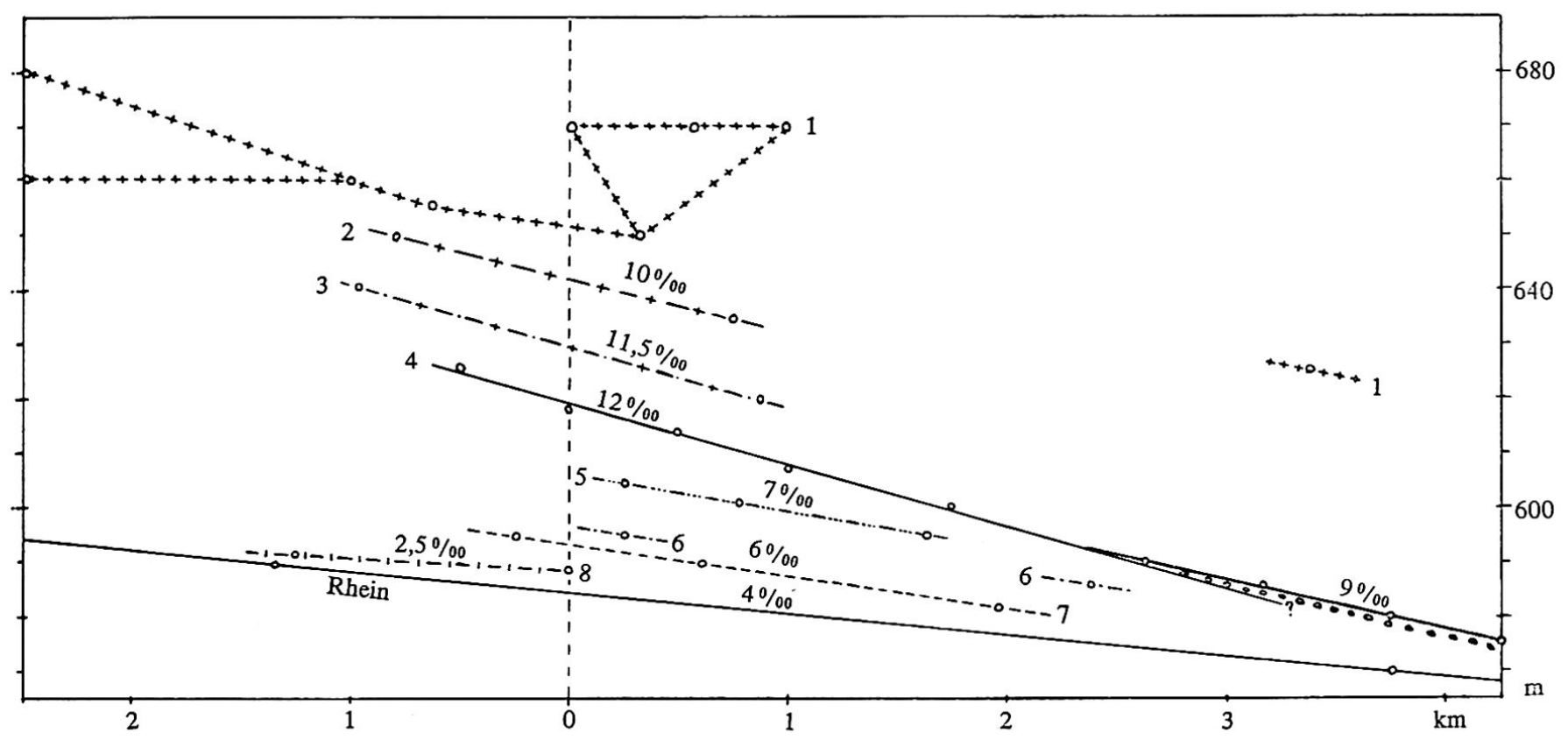

Figur 6. Höhenlage und Gefälle der verschiedenen Rheinterrassen zwischen Bonaduz und Ems. Die Ziffern bezeichnen die verschiedenen Niveaus: Erklärung im Text. Km O: Zusammenfluß von Vorder- und Hinterrhein

der Schwemmkegel der Plessur in Frage, welcher offenbar damals kräftig wuchs. Damit kommen wir in der Datierungsfrage weiter: Der letzte kräftige Klimarückschlag, der zu einer massiven Aufschüttung führen konnte, ist die jüngere Dryaszeit, hier hinein gehört also auch die Entstehung der Emser Terrasse als Folge der Aufschüttungen der Plessur.

5.-7. Zweite Serie von Erosionsniveaus. Flächenmäßig wenig bedeutend. Gefälle bis auf $6 \%$ abnehmend, das heißt die Terrassen werden talabwärts über das Haptniveau aufsteigen und damit von Erosions- zu Akkumulationsflächen, nämlich den Aufschüttungsebenen Richtung Bodensee. Die Erosionsbasis rückt also merklich in die Ferne, und der Fluß nimmt langsam die heutigen Charakteristika an.

8. Die Isla-Terrassen, Akkumulationsterrassen am untersten Hinterrhein; Gefälle nur 2,5\%o. Die große Breite der Terrasse NNE Bonaduz weist auf starke Seitenerosion und diese wiederum auf einen Aufstau hin. Es scheint, daß der Vorderrhein während einiger Zeit beträchtlich mehr Schutt führte und sein Bett etwa $3 \mathrm{~m}$ hoch anfüllte, was dem Hinterrhein das Gefälle nahm. Als Ursache ist kein Klimawechsel anzunehmen; vielmehr dürfte eine zu große Schuttzufuhr von den Hängen der Schlucht im Flimser Bergsturz angenommen werden.

Heutiges Flußniveau: Das Gefälle ist merklich kleiner als früher und liegt bei 4-5\%. Bei Hochwasser wird trotzdem Schutt von einer Körnung transportiert, die jener der hohen Terrassen kaum nachsteht. Die Erosionsleistungen sind relativ ge- ring, so daß die Vegetation ihre Spuren zu verdekken vermag.

Rückblickend fällt das außerordentlich hohe Gefälle des Hauptniveaus von Ems auf. Ein solcher Talboden kann unmöglich unter heutigen klimatischen Verhältnissen entstehen. Die Seitenerosion war sehr aktiv, und das bei einem Gefälle, dem heute eine kräftige Tiefenerosion folgen würde. Also war der Belastungsgrad des Flusses damals viel höher: entweder mehr Schutt oder weniger Wasser als heute, oder beides. Solche Verhältnisse kennen wir aber nur bis zur Wende Pleistozän/Holozän, das heißt etwa 7000 oder 8000 v. Chr.

Einen Anhaltspunkt dafür liefern nun auch die Schwemmkegel der südlichen Talseite: Uberall stellen wir fest, daß nur die obersten Schwemmassen (2-4 m) warmzeitliche Schnecken enthalten, die tieferen aber (nach den bisherigen Funden) nicht; also ist ihre Aufschwemmung durch Feinmaterial mit der Klimabesserung (Boreal) zum Abschluß gekommen. Trotz des feinen lockeren Materials sind am Fuß der Kegel aber nur relativ kleine Unterschneidungen (Abb. 2) zu sehen, was bedeutet, $\mathrm{da} ß$ der Rhein sich fast gleichzeitig in seiner heutigen Lage fixiert hat.

Die Rheinterrassen westlich von Chur erweisen sich damit als Schlüsselpunkt für das Verständnis spätglazialer morphogenetischer Abläufe.

\section{Zusammenfassung}

Aus den vielen Einzelangaben der vorangehenden Kapitel läßt sich ein recht präziser Ablauf der spät- 
glazialen Morphogenese des Untersuchungsgebietes festhalten. Neue Funde können allerdings Verschiedenes wieder in Frage stellen, doch werden diese Ergebnisse als Diskussionsbeitrag hoffentlich einen Dienst erweisen. Entsprechend sind aber vor allem die zeitlichen Zuordnungen zu verstehen.

Bölling-Interstadial: Gebiet eisfrei, eventuell RheinLinth-See bis hierher reichend. Niedergang verschiedener Bergstürze, die ersten vielleicht noch auf Toteis; dazwischen lokale fluviatile Erosion und Akkumulation. Geländeoberfläche oberhalb Chur am Ende höher als heute.

Altere Dryaszeit: Vorstoß des Vorderrheingletschers (zuerst eventuell Randlagen im Gebiet der heutigen Emser Werke, dadurch Ablenkung des Hinterrheins $S$ Ils Aults mit mehreren Verlegungen der randglazialen Rinne, daher heute die unpassend quer zum Tal verlaufenden Tomas).

Churer Stadium: Auskolkung einer vorhandenen Mulde im Bergsturzschutt zum Becken von Bonaduz, relative Schonung des Riegels der "Aults», Abtrag größerer Mengen an Bergsturzschutt von hier bis nach Chur und damit Vorbereitung der heutigen Tomalandschaft.

Toteisphase: Bildung des großen Kames S Reichenau und ähnlicher kleinerer Schotter.

Frühes Alleröd: Entstehung der obern Serie von Erosionsterrassen des Rheins zwischen Bonaduz und Tamins durch Senkung der lokalen Erosionsbasis; Ablösung der Eisschwelle durch eine Bergsturztrümmerschwelle.

Alleröd: Wahrscheinlich Eintiefung des Rheins unter die Emser Terrasse.

Jüngere Dryaszeit: Bildung riesiger Schwemmkegel aus dem Penninikum, Stau des Rheins durch den Plessurschuttkegel, Bildung der Emser Terrasse, Unterschneidung der Tomahänge.

Präboreal: Abschluß der Schüttungen, letzte Unterschneidungen der Tomas und der Schwemmkegel durch den Rhein. Einwanderung einer wärmeliebenden Schneckenfauna. Bildung der zweiten Gruppe von Erosionsterrassen am Rhein, Fixierung seines Laufes.

Seither: Bildung der Isla-Terrassen am Hinterrhein durch Aufschüttung des Vorderrheins in seinem Bett, Ursache lokal. Wiedereintiefung von Vorderund Hinterrhein. Murgänge überschüitten die Schwemmkegel. Im übrigen relativ geringe morphologische Aktivität.

\section{Anmerkung}

1 Für die Bestimmung der Bruchstücke sei an dieser Stelle Herrn Dr. L. Forcart (Basel) unser bester Dank ausgesprochen.

\section{Literatur}

Gouda G. H. (1962): Untersuchungen an Lößen der Nordschweiz. Geogr. Helvet. 1962, 133-220. Nabholz W. K. (1954): Neue Beobachtungen im Bergsturzgebiet S Reichenau-Tamins. Verh. Naturwiss. Ges. Basel 65/1, 67-81.

Pavoni N. (1968): Über die Entstehung der Kiesmassen im Bergsturzgebiet von Bonaduz-Reichenau. Ecl. Geol. Helv. 61/2, 494-499.

Remenyik T. (1959): Geologische Untersuchungen der Bergsturzlandschaft zwischen Chur und Rodels. Ecl. Geol. Helv. 52/1, 170-234.

Stehlin H. G. (1941): Eine interessante Phase in den Wandlungen unserer pleistozänen Säugetieffauna. Ecl. Geol. Helv.. 34/2, 287-291.

Wyß R. (1968): Das Mesolithikum. In: Ur- und Frühgeschichtliche Archäologie der Schweiz, Bd. 1. Verlag Schweiz. Ges. für Ur- und Frühgeschichte, Basel 1969, 123-144.

Zimmermann H.W. (1963): Die Eiszeit im westlichen zentralen Mittelland. Mitt. Naturf. Ges. Solothurn, Heft 21.

\section{Résumé}

A plusieurs points de vue, de nouvelles investigations sur la morphogenèse des «tomas» de DomatEms (Grisons) ont mené à des résultats intéressants: Dans les couches superficielles des grands cônes de déjection de la vallée du Rhin, on trouve une faune de mollusques qui semble représenter la grande amélioration climatique postglaciaire d'environs 7800 av. J.-C.

Sur les tomas d'Ems et à leurs flancs, de petits dépôts fluviaux inattendus sont interprétés comme des formations glacio-marginales.

Dans la grande gravière de Reichenau, on peut démontrer la présence du glacier rhénan et son travail érosif bien antérieurement à la déposition des cailloutis de Bonaduz; ceux-ci remplissent donc un bassin partiellement creusé par ce même glacier dans les débris d'éboulements déposés de Flims à Ems.

Les terrasses fluviales longeant le Rhin de Bonaduz à Ems sont caractérisées par une inclinaison changeante d'une manière typique, ce qui permet des conclusions sur les bases locales d'érosion.

La combinaison de ces résultats nous mène à une fixation nouvelle de l'ordre chronologique des événements d'importance morphogénétique à l'ouest de Coire. 